\title{
Post mastectomy chestwall irradiation of right sided breast cancer with single energy electron beam Vs three dimensional conformal radiotherapy with photons - Analysis of dosimetric parameters.
}

\author{
${ }^{1}$ Subbarao Nukasani, ${ }^{2}$ Kesava Ramgopal A, ${ }^{3}$ Subbarayudu S, ${ }^{4}$ Raghavendra VK, \\ ${ }^{5}$ Jagannatharaonaidu K V \\ (Department of Radiation Oncology, NRI medical college, India)
}

\begin{abstract}
The Post mastectomy chestwall radiation is routinely delivered using tangential photon fields. However underlying lung is a major organ at risk and this report studies the use of single electron beam radiotherapy in these patients. To compare mean left lung dose, percentage volume of left lung receiving dose more than 20Gy. (V20Gy), percentage volume of planned target volume (PTV) receiving more than 95\% of prescribed dose (V95\%). There was significant difference in favour of electron-beam plans for mean lung dose $(p=0.004), V 20 G y$ of left lung $(p=0.003)$ and there was no significant difference in mean heart dose. The difference in percentage volumeof PTV receiving more than 95\% of prescribed dose $(p=0.069)$ showed trend towards significance.
\end{abstract}

Keywords: Chestwall radiotherapy, electrons.

\section{Introduction}

Modified radical mastectomy(MRM) remains the most-accepted surgical modality in operable breast cancer [1]. Three randomized clinical trials have shown that a disease-free and overall survival advantage is conferred by the addition of chest wall and regional lymph node irradiation in women with positive axillary lymph nodes after MRM [2-4]. Conventional post-mastectomy radiation therapy (PMRT) is often delivered with traditional field borders for chest wall and regional nodes. Although chest wall and regional nodes delineation techniques have been discussed with available contouring guidelines, computed tomography (CT)-based planning to treat chest wall and nodal regions as a whole PTV has been adopted into routine practice. We herein discuss the dosimetric parameter comparison between electron beam therapy and conventional tangential two field radiation in left sided chestwall in postmastectomy cases.

\section{Materials And Methods}

Eligibility criteria included: age $\geq 18$ years with operable breast cancer without evidence of distant metastasis (negative results on chest CT scans, abdomen and pelvis Ultrasound); resection of all gross disease by MRM with level I to II axillary dissection; negative surgical margins; Eastern CooperativeOncology Group performance score of $0-1$; completion of adjuvant chemotherapy; and no previous thoracic RT. Patients with serious comorbid diseases, such aschronic obstructive pulmonary disease, connective tissue disease, postoperative wound infections, and delayed wound healing, etc., that would have negatively affected their tolerance to radiation-induced skin or lung toxicity were not eligible. Patients with synchronous bilateral breast cancers were eligible. All patients provided written informed consent.before simulation, the patient was placed supine on a commercially available breast tilt board (Med-Tech 350) to make sternum parallel to the table, with both armsfully abducted (90 degrees or greater) and externally rotated, and head position secured. A planning CT scan at 5-mm intervals from mid-neck to diaphragm with no contrast enhancement was obtained for each patient using an AcQsim CT simulator (Philips Medical Systems).

At simulation, the mastectomy scar was routinely wired with radiopaque markers.Contours definition. The clinical target volumes (CTV) were defined to consist of ipsilateral chest wall, mastectomy scar for each patient. Treatment of internal mammary nodes (IMN) was strongly considered when primary tumor was located in central or medial part of the breast. Each CTV was delineated according to the breast cancer atlas for radiation therapy planning consensus definitions of the Radiation Therapy Oncology Group (RTOG). The chest wall CTV was expanded $1 \mathrm{~cm}$ to become chest wall PTV, except that anterior, posterior and cranial borders were unchanged. This modification was made mainly to account for build-up region or spare underling normal lung from high dose radiation [5,6]. If IMN irradiation not indicated, medial border of chest wall PTV was usually at medial edge of sternal-rib junction (with reference to medial border of contralateral breast). The anatomically based guidelines established by Dijkema et al. [7] were also referenced when regional node CTVs 
were contoured. Generally, expansions of $5 \mathrm{~mm}$ to CTV for supra/infraclavicular nodes and $7 \mathrm{~mm}$ to CTV for IMN were made to form the PTV for supra/infraclavicular nodes and IMN, respectively.If IMN included, PTV for IMN would be smoothly integrated into PTV for chest wall. The PTVfor supra/infraclavicular nodes would match the PTV for chest wall, and IMN if indicated, at the clavicle head inferiorly.As a result, a whole PTV including both chestwall and regional nodes formed. The organs at risk (OARs) surrounding the targets, including bilateral lungs, heart, contralateral breast, ipsilateral humeral head, spinal cord, and esophagus, werecontoured as well. The heart was defined as from its apex to the junction of great vessels with myocardium, and esophagus was contoured from cricoid cartilage to cardia.In addition, the healthy tissue was defined as the patient's volume covered by the CT scan minus the envelope of the PTV to account for the spillage of prescription dose.

Three dimensional conformal radiotherapy plans were generated using 6MV medial and lateral tangential beams with paired wedges and $0.5 \mathrm{~cm}$ bolus, multileaf collimators were used to shape the beams. Electron-beam plans were generated with a single anterior oblique beam of $12 \mathrm{MeV}$ and $0.5 \mathrm{~cm}$ bolus. A dose of 50 Gy was prescribed to PTV in 2 Gy per fraction, 25 fractions. Dose plans were evaluated and data analysis was done using Windows SPSS software for Statistical analysis version 20.

\section{Results}

There was significant difference in favour of electron-beam plans for mean lung dose $(\mathrm{p}=0.004)$, V20Gy of left lung $(p=0.003)$. There was no significant difference in mean heart dose $(p=0.624)$. The difference in percentage volume of PTV receiving more than $95 \%$ of prescribed dose $(\mathrm{p}=0.069)$ showed trend towards significance.

\section{Discussion}

The rationale for post-mastectomy radiotherapy is to prevent local recurrence in the chest wall, skin , mastectomy scar and regional lymph nodes. Complexity of shape and position of the chest wall, makes it necessary to pay careful attention to daily positioning and accurate treatment delivery. Electrons differ from photons in that electrons have a finite range; electrons travel a certain distance and then stop, and their kinetic energy is zero. The one difference in the geometry is that an electron cone is used that requires fixed collimator positions in length and width. Although electron beams have some of the same features as photon beams, their finite range and the presence of bremsstrahlung contamination are unique. Field size, flatness and symmetry, and sharpness of the beam edge for electrons can be measured similarly as for photon beams, however, the measurement depth is typically at $\mathrm{dm}$ or at the $95 \%$ dose level beyond $\mathrm{dm}$. Mean lung dose was found to be significantly less with electron beam theapy using single energy electron therapy. As only a small amount of electron dose reaches the lung, the mean dose received by lung is very low this electron beam therapy. Volume of lung receiving a dose more than $20 \mathrm{~Gy}$ was also significantly less with electron therapy. However there was no difference in percentage volume of PTV receiving more than $95 \%$ dose.

\section{Conclusion}

Single energy electron-beam therapy can significantly reduce doses to heart and left lung in postmastectomy radiotherapy of left sided breast tumors with appropriate patient selection.

\section{References}

[1]. Yu KD, Di GH, Wu J, Lu JS, Shen KW, Shen ZZ, Shao ZM: Development and trends of surgical modalities for breast cancer in China: a review of 16-year data. Ann Surg Oncol 2007, 14(9):2502-2509.

[2]. Overgaard M, Hansen PS, Overgaard J, Rose C, Andersson M, Bach F, Kjaer M, Gadeberg CC, Mouridsen HT, Jensen MB, et al: Postoperative radiotherapy in high-risk premenopausal women with breast cancer who receive adjuvant chemotherapy. Danish Breast Cancer Cooperative Group 82b Trial. N Engl J Med 1997, 337(14):949-955.

[3]. Recht A, Gray R, Davidson NE, Fowble BL, Solin LJ, Cummings FJ, Falkson G, Falkson HC, Taylor SG, Tormey DC: Locoregional failure 10 years after mastectomy and adjuvant chemotherapy with or without tamoxifen without irradiation: experience of the Eastern Cooperative Oncology Group. J Clin Oncol 1999, 17(6):1689-1700.

[4]. Ragaz J, Olivotto IA, Spinelli JJ, Phillips N, Jackson SM, Wilson KS, KnowlingMA, Coppin CM, Weir L, Gelmon K, et al: Locoregional radiation therapy in patients with high-risk breast cancer receiving adjuvant chemotherapy: 20-year results of the British Columbia randomized trial. J Natl Cancer Inst2005, 97(2):116-126.

[5]. Rudat V, Alaradi AA, Mohamed A, Ai-Yahya K, Altuwaijri S: Tangential beam IMRT versus tangential beam 3D-CRT of the chest wall in postmastectomy breast cancer patients: a dosimetric comparison. Radiat Oncol 2011, 6:26.

[6]. Fontanilla HP, Woodward WA, Lindberg ME, Kanke JE, Arora G, Durbin RR, Yu T-K, Zhang L, Sharp HJ, Strom EA, et al: Current clinical coverage of Radiation Therapy Oncology Group-defined target volumes for postmastectomy radiation therapy. Practical Radiation Oncology 2012, 2(3):201-209.

[7]. Dijkema IM, Hofman P, Raaijmakers CP, Lagendijk JJ, Battermann JJ, Hillen B: Loco-regional conformal radiotherapy of the breast: delineation of the regional lymph node clinical target volumes in treatment position. Radiother Oncol 2004, 71(3):287-295. 